\title{
Muon content of ultra-high-energy air showers: Yakutsk data versus simulations
}

\author{
A.V. Glushkov, I.T. Makarov, M.I. Pravdin, I.E. Sleptsov, \\ (The Yakutsk EAS Array) \\ Yu.G. Shafer Institute of Cosmophysical Research and Aeronomy, Yakutsk 677980, Russia \\ D.S. Gorbunov, G.I. Rubtsov, S.V. Troitsky \\ Institute for Nuclear Research of the Russian Academy of Sciences, \\ 60th October Anniversary Prospect 7a, Moscow 117312 Russia
}

\begin{abstract}
We analyse a sample of 33 extensive air showers (EAS) with estimated primary energies above $2 \cdot 10^{19} \mathrm{eV}$ and high-quality muon data recorded by the Yakutsk EAS array. We compare, event-by-event, the observed muon density to that expected from CORSIKA simulations for primary protons and iron, using SIBYLL and EPOS hadronic interaction models. The study suggests the presence of two distinct hadronic components, "light" and "heavy". Simulations with EPOS are in a good agreement with the expected composition in which the light component corresponds to protons and the heavy component to iron-like nuclei. With SYBILL, simulated muon densities for iron primaries are a factor of $\sim 1.5$ less than those observed for the heavy component, for the same electromagnetic signal. Assuming two-component proton-iron composition and the EPOS model, the fraction of protons with energies $E>10^{19} \mathrm{eV}$ is $0.52_{-0.20}^{+0.19}$ at $95 \%$ confidence level.
\end{abstract}

Number of muons in extensive air showers (EAS) is used as an estimator of the primary composition of ultra-high-energy cosmic rays detected by surface arrays. Precision of muon-based composition studies is limited by their sensitivity to hadronic interaction models which incorporate extrapolation of experimental data to kinematic regions never tested in a laboratory experiment. On the other hand, the responses of the ground-based cosmic-ray detectors to different EAS components are different, and variations in the muon content may affect the relation between the signal recorded on the ground and the inferred energy 
of the primary particle. This is important in particular for detectors sensitive to the muon component (e.g. the surface detector of the Pierre Auger Observatory).

In this Letter we compare the observed and simulated muon contents of EAS detected by the Yakutsk array [1. This experiment is currently the only one capable of detecting air showers initiated by particles of $E \gtrsim 10^{19} \mathrm{eV}$ and equipped with muon detectors. Large area and high saturation threshold of each detector station make it possible to obtain high-quality muon data [2].

Recently, the Pierre Auger Observatory (PAO) collaboration reported [3] an excess of muons as compared to simulations with the QGSJET II hadronic interaction model [4]. Since PAO is not equipped with muon detectors, indirect methods were used. The same result has been previously reported [5] by the Yakutsk collaboration (by making use of older hadronic models). Here, we perform a direct and detailed study of this effect for each individual shower in a high-quality sample and for the sample as a whole, in the frameworks of two different hadronic interaction models, SIBYLL [6] and EPOS [7] (the muon content of showers simulated with QGSJET II is between these two [8]). We use a precise statistical method [9] to construct the distributions of muon densities simulated for individual events, which are then compared to the observed data (see Refs. [10, 11] for other applications of the method).

In our study, we use a sample of 33 events with reconstructed energies above $2 \cdot 10^{19} \mathrm{eV}$, zenith angles up to $45^{\circ}$, core location inside the array and high-quality muon data recorded. The latter criterion means that we require at least three operating muon detectors at distances between $400 \mathrm{~m}$ and $2000 \mathrm{~m}$ from the shower axis, which allows one to reconstruct the lateral distribution of the muon density. For each of the events, we simulated a library of showers with different primary energies but with the same arrival direction as observed. The simulations were performed with CORSIKA 6.611 [12] using FLUKA 2006.3 [13] as a low-energy hadronic interaction model and either SIBYLL 2.1 [6] or EPOS 1.61 [7] as a high-energy model. For SIBYLL, multisampling $\left(20 \times 10^{-4}\right)$ was used to suppress artificial fluctuations due to thinning [14]. For the case of EPOS, we used thinning $\left(10^{-5}\right)$ with weights limitations [15] to save computational time; more artificial fluctuations are expected in this case. The responce of the scintillators was simulated with GEANT in Ref. [16].

The Yakutsk collaboration uses the signal density at $600 \mathrm{~m}$ from the shower core, $S(600)$, to estimate the energy $E_{0}$ of the primary particle [17]. The relation between $S(600)$ and $E_{0}$ was obtained by making use of the constant intensity cuts method (to relate $S(600)$ in inclined and vertical showers) and of the calibration by Cherenkov light (to relate $S(600)$ and 

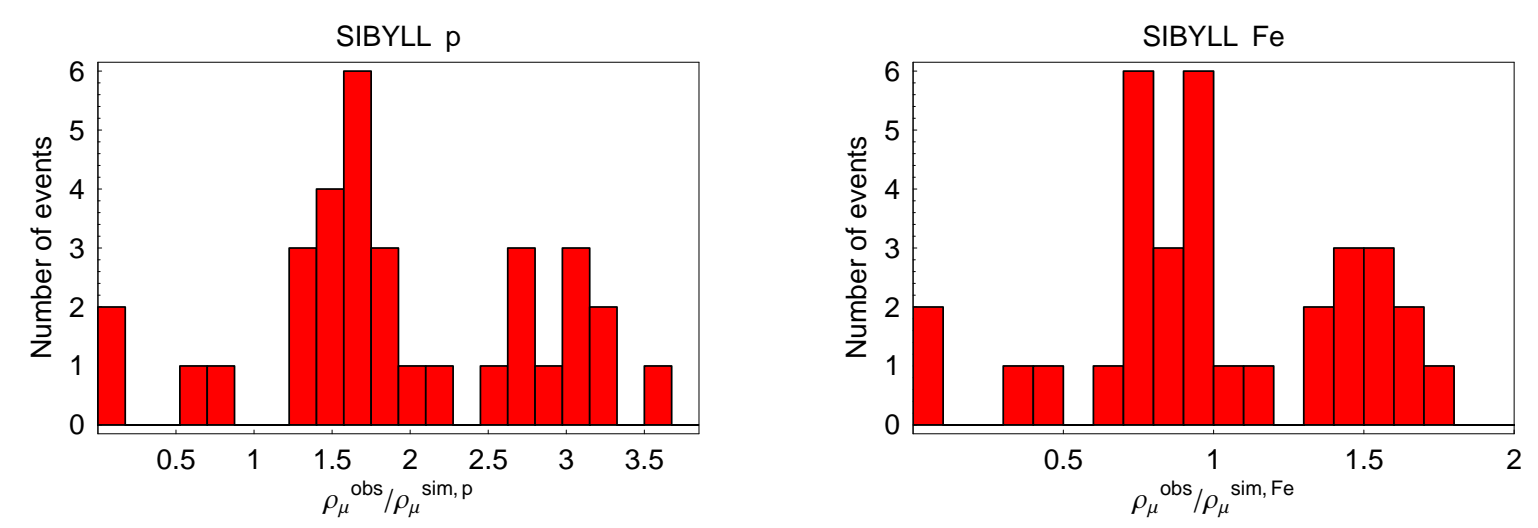

Figure 1: Distributions of $\eta_{\mathrm{p}}$ and $\eta_{\mathrm{Fe}}$ with SIBYLL.

$E_{0}$ for vertical showers). In this study, however, we do not use the estimated energy and hence do not use these relations; instead, we use $S(600)$ measured in individual showers to compare real and simulated events. To obtain the value of $S(600)$ in simulated showers, we fit the lateral distribution function with the same formula [17] as used in processing of real data. We use the muon density at $1000 \mathrm{~m}$ from the shower axis, $\rho_{\mu}(1000)$, as the estimator of the muon content.

Making use of the method described in detail in Ref. [9], we selected simulated showers whose $S(600)$ are consistent with the observed one taking into account experimental errors in the determination of this parameter, and obtained the probability distribution $f\left(\rho_{\mu}^{\mathrm{sim}}\right)$ to have $\rho_{\mu}(1000)=\rho_{\mu}^{\text {sim }}$ in a simulated shower arriving from the same direction and having the same $S(600)$ as the real one. This procedure was repeated for each event for assumed proton and iron primaries. We used from 400 to 1000 simulated showers for each observed event depending on its zenith angle. These distributions were analyzed to estimate the ratios

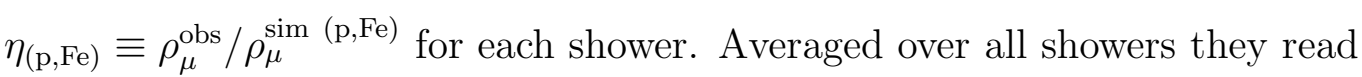

$$
\begin{array}{rll}
\text { SIBYLL : } & \eta_{\mathrm{p}}=1.92_{-0.10}^{+0.24}, & \eta_{\mathrm{Fe}}=1.00_{-0.04}^{+0.09}, \\
\text { EPOS : } & \eta_{\mathrm{p}}=1.14_{-0.06}^{+0.16}, & \eta_{\mathrm{Fe}}=0.66_{-0.03}^{+0.06}
\end{array}
$$

(only statistical errors are shown). The average composition is therefore iron-like assuming the SIBYLL interaction model and slightly heavier than protons assuming EPOS.

However, a glance at the distribution of $\eta$ calculated event-by-event (Figs. 1, 21) suggests that averaging is not a proper way to interpret the data which are actually trimodal.

The two (almost) muonless events may be interepreted as photons [11]; here we concentrate on the rest of the sample. One is tempted to interpret two other peaks as a signature

\footnotetext{
${ }^{1}$ Except for the determination of the data set.
} 

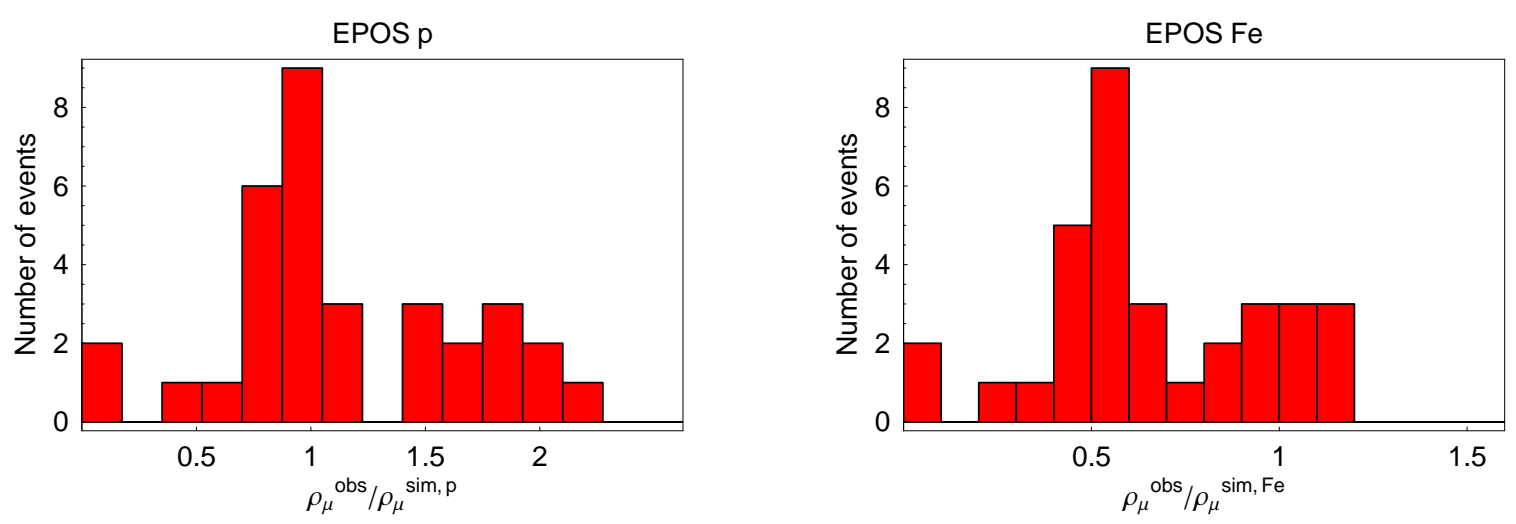

Figure 2: Distributions of $\eta_{\mathrm{p}}$ and $\eta_{\mathrm{Fe}}$ with EPOS.

of the mixed composition with the low-muon-density peak corresponding to protons and the second peak corresponding to heavy nuclei. As we will discuss elsewhere, this picture is indeed expected for UHECRs of astrophysical origin because the mean free path of intermediate-mass nuclei in the bath of cosmic background radiation is considerably shorter, see for instance Ref. [18].

The intuitive requirement that the cosmic-ray hadrons are stable nuclei with atomic numbers $1 \leq Z \leq 56$ appears inconsistent with the results of the SYBILL simulations (see Fig. 1). Following Ref. [3], one has to assume a multiplicative correction factor for the simulated muon density which should be about 1.5 to satisfy this requirement. On the other hand, results of the EPOS simulations agree with expectations within statistical uncertainties. Our results are based on the simulation of both the signal density $S(600)$ and the muon density $\rho_{\mu}(1000)$ and are thus sensitive not only to the hadronic model but also to the model of electromagnetic interactions (EGS4 [19]). In principle, systematic underestimation of the electromagnetic signal by EGS4 may explain the SYBILL result (then EPOS simulates too high muon densities). Here, we accept the option that both EGS4 and EPOS are correct and do not consider the sample of showers simulated with SIBYLL in the rest of the study.

The distribution of muon densities of simulated showers presented in Fig. 2 clearly indicates the presence of heavy nuclei in UHECRs. The limited statistics of our sample gives no chance to distinguish imprints of intermediate mass nuclei, e.g. CNO, and we leave this problem for future. The present sample is fully consistent with purely three-component (photons, protons and iron) primary composition. This fact is illustrated in Fig. 3, which demonstrates that most of our 33 events can be consistently identified with photon, proton or iron primary. 


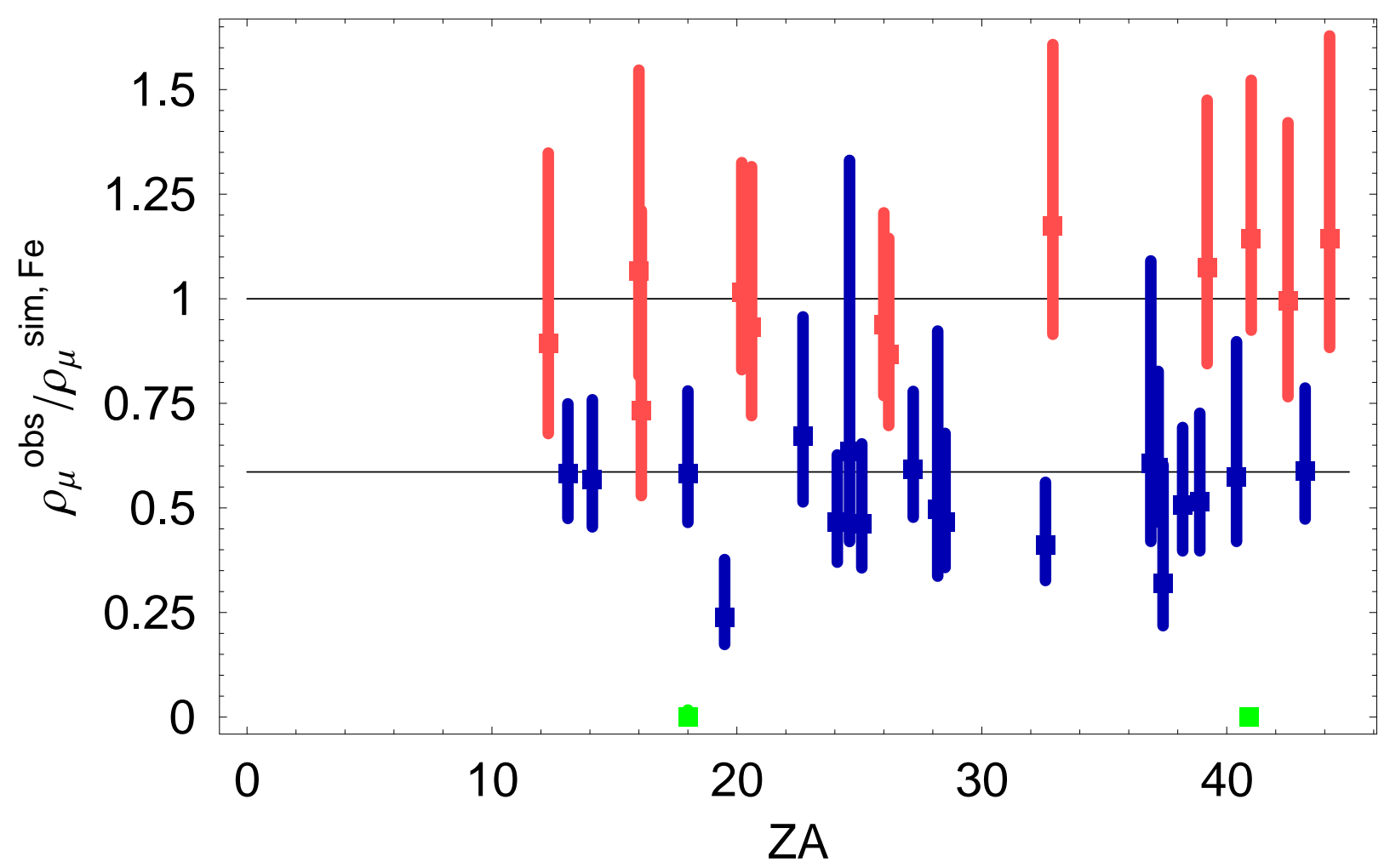

Figure 3: The ratios $\eta^{\mathrm{Fe}}=\rho_{\mu}^{\mathrm{obs}} / \rho_{\mu}^{\mathrm{sim}, \mathrm{Fe}}$ of observed and simulated muon densities at $1000 \mathrm{~m}$ from the shower core versus the zenith angle. Central values and $68 \%$ CL error bars (both statistical and systematic errors are included) were obtained for each event from the actual distributions of iron-induced showers simulated with the EPOS hadronic model for the arrival direction and $S(600)$ consistent with the observed ones. The error bars include the precision of reconstruction of $S(600)$ and $\rho_{\mu}(1000)$ as well as intrinsic shower-to-shower fluctuations. Two muonless events $\left(\eta^{\mathrm{Fe}}=0\right)$ are shown in light green; the rest of events are separated into two groups, proton-like (dark blue) and iron-like (light pink), based on event-by-event probabilities calculated as in Ref. [9]. The horisontal lines represent expected average values of $\eta^{\mathrm{Fe}}$ for iron (1.00) and protons (0.59). 
One may estimate quantitatively the significance of bimodality seen in the distribution of $\eta$, Figs. 1, 2, for all events except muonless ones. The simplest measure of bimodality is the kurtosis $c_{4}$, the 4 th moment of a distribution. A slightly better measure suitable for non-symmetric distributions is the so-called bimodality coefficient $b=\left(1+c_{3}^{2}\right) /\left(c_{4}+3\right)$, where $c_{3}$ is the skewness (the 3rd moment) of a distribution and we use the definition of $c_{4}$ which provides $c_{4}=0$ for the normal distribution. We simulated 10000 sets, each of 31 numbers drawn from a normal distribution with the same mean and standard deviation as in the real data for $\eta=\rho_{\mu}^{\text {(obs) }} / \rho_{\mu}^{(\operatorname{sim})}$ and estimated the fraction of simulated sets which have the same or stronger bimodality than the data, using both $c_{4}$ and $b$. This fraction amounts to a few per cent (the precise number depends on the interaction model and assumed primary used to calculate $\rho_{\mu}^{(\operatorname{sim})}$, as well as on the estimator, $c_{4}$ or $b$; the typical numbers are 0.03 to 0.05$)$. We therefore conclude that the bimodality is confirmed at the $95 \%$ confidence level.

As discussed in detail in Ref. [9], the method we use is not sensitive to the relation between $S(600)$ and energy used for the experimental energy determination: simulated showers are selected by the value of $S(600)$. Thus a sample of showers with reconstructed energies $E>2 \cdot 10^{19} \mathrm{eV}$ may be used to constrain the primary composition for a different energy range, related to $S(600)$ by simulations. Motivated by the fact that a given $S(600)$ corresponds to a lower energy of a primary of a shower simulated with EPOS as compared to the Yakutsk energy reconstruction procedure (more details will be discussed elsewhere), we use our sample to infer primary composition at $E>10^{19} \mathrm{eV}$. The correction for the possible incompleteness of the sample determined by a cut in the reconstructed energy ("lost particles", see Ref. [9] for details) is taken into account in the results presented below. Systematic uncertainties of the approach have been discussed in Refs. [9, 10, 11.

Following the procedure of Ref. [9], we estimate the proton and iron fractions in the integral flux of cosmic rays at $E>10^{19} \mathrm{eV}$ assuming two-component proton-iron composition and the EPOS interaction model. The most probable proton fraction is $\epsilon_{p} \approx 0.52$ with the allowed interval

$$
0.32 \leq \epsilon_{\mathrm{p}} \leq 0.71 \quad(95 \% \mathrm{CL}), \quad E>10^{19} \mathrm{eV}
$$

which corresponds to the fraction of the heavy (iron-like) component

$$
0.29 \leq \epsilon_{\mathrm{Fe}} \leq 0.68 \quad(95 \% \mathrm{CL}), \quad E>10^{19} \mathrm{eV}
$$

These results are in a good agreement with the results [20] of hybrid observations at PAO and in a worse agreement with the results [21] of HiRes. 
One may in principle try to distinguish between various possible reasons of the discrepancies between simulated (with SYBILL in our case) and observed muon densities. In particular, incorrect extrapolation of the normalization of the cross section and/or multiplicity of hadronic interactions in a simulation code would probably result in a discrepancy in the total muon number of a shower while problems with the distribution of transverse momenta may show themselves in deviation of the observed muon lateral distribution function (LDF) from the simulated one. A more detailed study is in progress which will include the analysis of signals at each particular detector without fitting LDF by any predetermined function, the study of the QGSJET hadronic model and the analysis of the depth of the maximal shower development expected from the present results. The sample of events will be supplemented by inclined showers.

As may be inferred from Fig. 1 of Ref. [3] and from the discussion there, the energies of iron-like nuclei would on average be estimated by the PAO surface detector by some $15 \%$ higher than the energies of protons, for the same true energy of the primary. Given the steeply falling UHECR spectrum and assuming the mixed composition favoured by the present study, the samples of events with reconstructed energies higher than a fixed one would be biased towards heavy primaries. This may explain the results of Ref. [3] pointing to a somewhat stronger underestimation of average muon content by simulations than indicated by the results, Eqs. (1), (2), of the present work.

To summarize, we compared the observed muon densities of UHE air showers with those simulated with different hadronic models and for different primaries. The mixed primary composition was resolved, on an event-by-event basis, into two distinct, light (proton-like) and heavy (iron-like) components. The results reported here, namely the presence of the heavy and light components in the UHECR flux, consistency of EPOS and failure of SIBYLL to reproduce correctly the muon content of air showers, may have substantial consequences for understanding the origin of UHECR and the physics of high-energy hadron collisions, as well as for the energy estimation by surface detectors sensitive to muons (currently operating Pierre Auger Observatory as well as SUGAR and Haverah Park arrays).

We are indebted to Yu. Bykov, V. Rubakov and I. Tkachev for helpful discussions. This work was supported in part by the Russian Foundation of Basic Research grants 07-0200820 (INR team), 05-02-17857a (the Yakutsk team), by the grants of the President of the Russian Federation NS-7293.2006.2 (government contract 02.445.11.7370; INR team), MK-2974.2006.2 (DG), NS-7514.2006.2 (government contract 02.120.11.7514; the Yakutsk team), by the Dynasty Foundation (GR) and by the Russian Science Support Foundation 
(ST). Numerical part of the work was performed at the computer cluster of the Theoretical Division of INR RAS.

\section{References}

[1] V. Egorova et al., Nucl. Phys. Proc. Suppl. 136, 3 (2004); B.N. Afanasiev et al., Proc. Tokyo Workshop on Techniques for the Study of Extremely High Energy Cosmic Rays (Tokyo), 1993, p. 35.

[2] A. V. Glushkov et al., JETP Lett. 71, 97 (2000).

[3] R. Engel et al. (Pierre Auger Collaboration), Proc. 30th ICRC (2007), arXiv:0706.1921 [astro-ph].

[4] S. Ostapchenko, Nucl. Phys. Proc. Suppl. 151, 143 (2006).

[5] A.V. Glushkov et al., Astropart. Phys. 4 (1995) 15; A.V. Glushkov et al., Phys. Atom. Nucl. 65 (2002) 1313.

[6] R. S. Fletcher, T. K. Gaisser, P. Lipari and T. Stanev, Phys. Rev. D 50, 5710 (1994).

[7] K. Werner, F. M. Liu and T. Pierog, Phys. Rev. C 74 (2006) 044902.

[8] T. Pierog and K. Werner, to appear in Proc. 30th ICRC (2007).

[9] D. S. Gorbunov, G. I. Rubtsov and S. V. Troitsky, Astropart. Phys. 28 (2007) 28.

[10] G. I. Rubtsov et al., Phys. Rev. D 73, 063009 (2006)

[11] A. V. Glushkov et al., JETP Lett. 85 (2007) 131.

[12] D. Heck et al., Report FZKA-6019 (1998), Forschungszentrum Karlsruhe.

[13] A. Ferrari, P. R. Sala, A. Fasso and J. Ranft, CERN-2005-010; A. Fasso, A. Ferrari, J. Ranft et al., eConf C0303241, MOMT005 (2003) arXiv:hep-ph/0306267.

[14] D. S. Gorbunov, G. I. Rubtsov and S. V. Troitsky, Phys. Rev. D 76 (2007) 043004.

[15] M. Kobal et al., Astropart. Phys., 15, 259 (2001).

[16] L. G. Dedenko et al., Nucl. Phys. Proc. Suppl. 136 (2004) 12. 
[17] A. V. Glushkov et al., Phys. Atom. Nucl. 63, 1477 (2000).

[18] D. Allard, E. Parizot, E. Khan, S. Goriely and A. V. Olinto, Astron. Astrophys. 443, L29 (2005).

[19] W. R. Nelson, H. Hirayama, D.W.O. Rogers, SLAC-0265 (permanently updated since 1985).

[20] M. Unger et al. (Pierre Auger Collaboration), Proc. 30th ICRC (2007), arXiv:0706.1495 [astro-ph].

[21] R. U. Abbasi et al. (The High Resolution Fly's Eye Collaboration), Astrophys. J. 622, 910 (2005). 